\title{
Metastatic Breast Cancer Presenting as Pulmonary Nodules and Right Axillary Lymph Node in a Male with Chronic Lymphocytic Leukemia
}

\author{
Ana S. Acostamadiedo Marx ${ }^{a, b} \quad$ Camilo Andrés Avendaño-Capriles ${ }^{a}$ \\ Julio A. Lemos ${ }^{c}$ Eric Gorman ${ }^{c}$ Jose M. Acostamadiedoc \\ aUniversidad Del Norte, Km.5 Vía Puerto Colombia, Barranquilla, Colombia; ${ }^{\circ}$ Fundación \\ Santa Fe de Bogotá, Hospital Universitario, Bogotá D.C, Colombia; 'FitzPatrick Cancer \\ Center, Champlain Valley Physicians Hospital, University of Vermont Health Network, \\ Plattsburgh, NY, USA
}

\author{
Keywords \\ Chronic lymphocytic leukemia - Male breast cancer - Secondary malignancy · Pulmonary \\ nodule $\cdot$ Occult breast cancer
}

\begin{abstract}
To our knowledge, this is the first case reported in the English literature of simultaneous occult male metastatic breast cancer presenting as pulmonary nodules and right axillary lymph node metastasis in a chronic lymphocytic leukemia (CLL) patient and is the second case of simultaneous male breast cancer and CLL reported. The first case was reported by Dubashi et al. [Curr Oncol. 2011;18(2):e101-2] in 2011. This unique clinical and pathological entity presents various challenges in its management, including early detection, screening, and treatment.
\end{abstract}

(C) 2021 The Author(s).

Published by S. Karger AG, Basel

\section{Introduction}

Chronic lymphocytic leukemia (CLL) has been linked to an increased incidence of secondary malignancies, like breast, thyroid, skin, lung, and prostate, also to an atypical morphology and aggressive presentations of these lesions. The previous can be explained by the alterations in humoral immunity caused by CLL [1]. We describe a case of simultaneous 
Fig. 1. Chest X-ray AP view demonstrates multiple small bilateral pulmonary nodules predominantly at the lung bases. The right hilum is prominent.

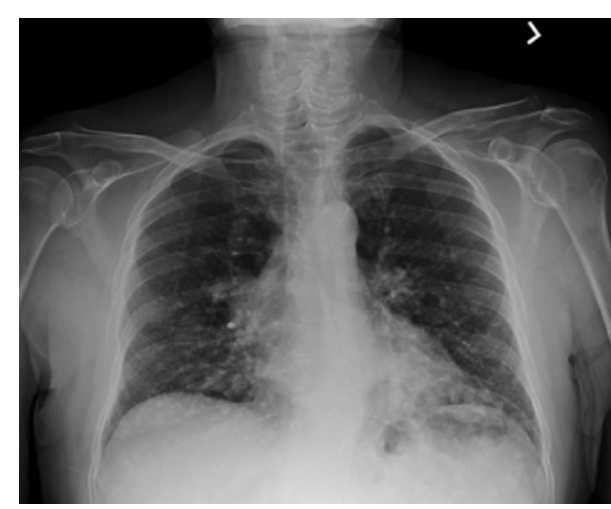

Fig. 2. Mass, right axillary fossa

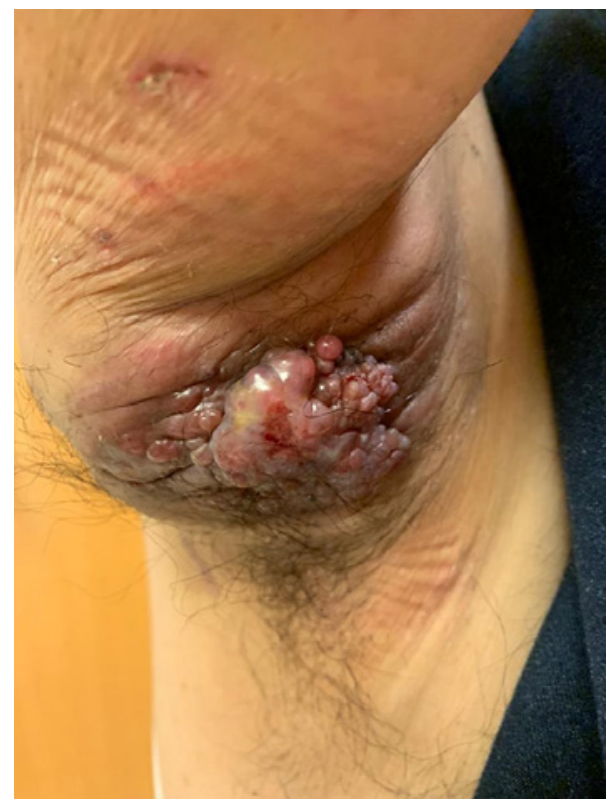

male metastatic breast cancer presenting as pulmonary nodules and right axillary mass in a CLL patient [2].

\section{Case Report}

A retired 71-year-old man with a previous clinical history of CLL in remission arrived to consult for follow-up after an upper respiratory tract infection diagnosis was made, for which a chest X-ray was ordered. AP view demonstrated multiple small bilateral pulmonary nodules, predominantly at the lung bases, and the right hilum, were prominent (Fig. 1). Eventually, due to the abnormal result, a CT scan was performed, which showed multiple pulmonary nodules, mediastinal adenopathy, and a proximal right humeral mass. The humeral nodule/mass was the size of a baseball, protruding from the right axillary fossa (Fig. 2) with some superficial skin ulceration and painless to palpation. The remainder of the physical examination was within normal limits, and the review of systems revealed no significant changes. Possible breast cancer symptoms like nipple secretions, retraction, or masses were interrogated and denied.

His most recent chemotherapy regimen for CLL was fludarabine, cyclophosphamide, and rituximab eightyears before. Relevant medical history besides CLL revealed rash, hyperlipidemia, 
Fig. 3. H\&E stain.

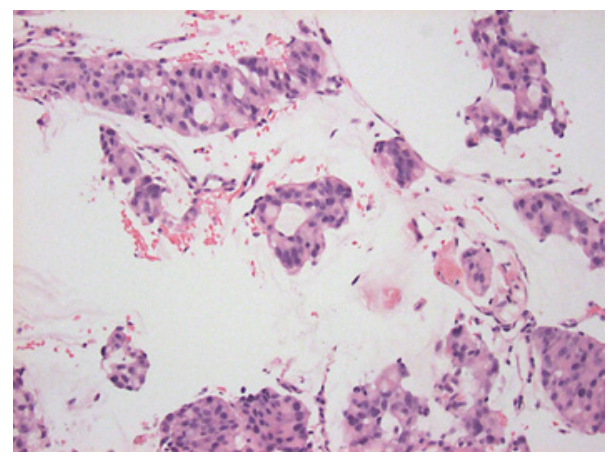

Fig. 4. PR stain.

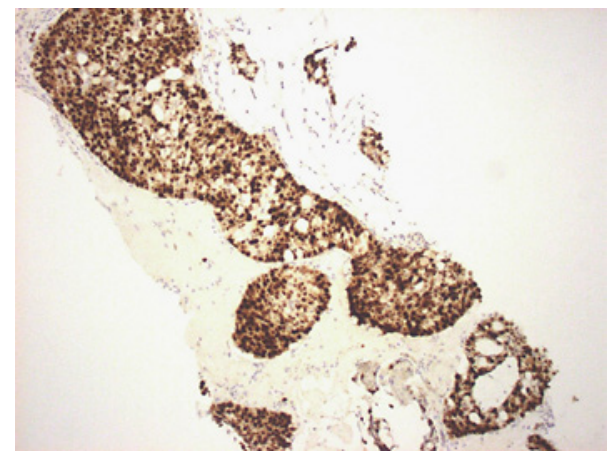

and iron and vitamin B12 deficiencies. His current medication regimen included aspirin $81 \mathrm{mg}$ tablet delayed release, calcium 600 + D (3), Diovan $80 \mathrm{mg}$ tablet, and ferrous sulfate $325 \mathrm{mg}$.

Two months before the visit, while in Mumbai, India, the patient felt the nodule, which was not ulcerated at the time. He consulted a physician, who told him the axillary lesion was likely Bartonellosis or "cat scratch" disease and advised him to wait for it to resolve spontaneously. A review of the scan and the prominent mediastinal lymphadenopathy raised the concern of CLL recurrence or Richter's transformation. Laboratory test results at the time revealed a slight anemia and thrombocytopenia $(\mathrm{WBC}=7.81 \mathrm{~K} / \mu \mathrm{L}, \mathrm{RBC}=3.91 \mathrm{M} / \mu \mathrm{L}$, $\mathrm{HGB}=12.4 \mathrm{~g} / \mathrm{dL}, \mathrm{HCT}=35.1 \%, \mathrm{MCV}=89.8 \mathrm{fL}, \mathrm{MCH}=31.7 \mathrm{pg}, \mathrm{MCHC}=35.3 \mathrm{~g} / \mathrm{dL}, \mathrm{RDW}=$ $13.3 \%$, Plat $=121 \mathrm{~K} / \mu \mathrm{L}$, and $\mathrm{MPV}=8.9 \mathrm{fL}$ ), findings that are not uncommon in patients treated with fludarabine.

A CT-guided right lower lobe lung biopsy was performed and revealed metastatic mucinous adenocarcinoma favoring breast origin. Ultrasound-guided core needle biopsy of the right axillary mass resulted in invasive ductal carcinoma with mucinous features, nuclear grade 2 with no definitive in situ component. The tumor was p53 and heavy chain myosin negative and estrogen receptor positive (ER+) and progesterone receptor positive (PgR+). H\&E, PR, and ER stains are shown (Fig. 3-5).

On CT, there were innumerable noncalcified pulmonary nodules within each lung. The largest pulmonary nodule present in the right upper lobe measured $1.6 \mathrm{~cm}$ in diameter. This pulmonary nodule and multiple additional pulmonary ones resulted in being hypermetabolic, with a maximum SUV of 8.3 (Fig. 6). There were multiple hypermetabolic mediastinal and bilateral hilar lymph nodes. An approximately $7-\mathrm{mm}$ right internal mammary lymph node was hypermetabolic. An about $4.5 \times 7 \mathrm{~cm}$ in diameter right axillary mass, the biopsy-proven metastatic breast cancer site, was hypermetabolic with a maximum SUV of 14.5 (Fig. 6). There was a small right pleural effusion. Symmetric retroareolar soft tissue density presented with the appearance suggesting gynecomastia. There was no associated 


\section{Case Reports in Oncology}

Fig. 5. ER stain.

\begin{tabular}{l|l}
\hline Case Rep Oncol 2021;14:1608-1615 \\
\hline DOI: 10.1159/000519744 & $\begin{array}{l}\text { @ 2021 The Author(s). Published by S. Karger AG, Basel } \\
\text { www.karger.com/cro }\end{array}$ \\
\hline
\end{tabular}

Acostamadiedo Marx et al.: Breast Cancer in a Male with CLL

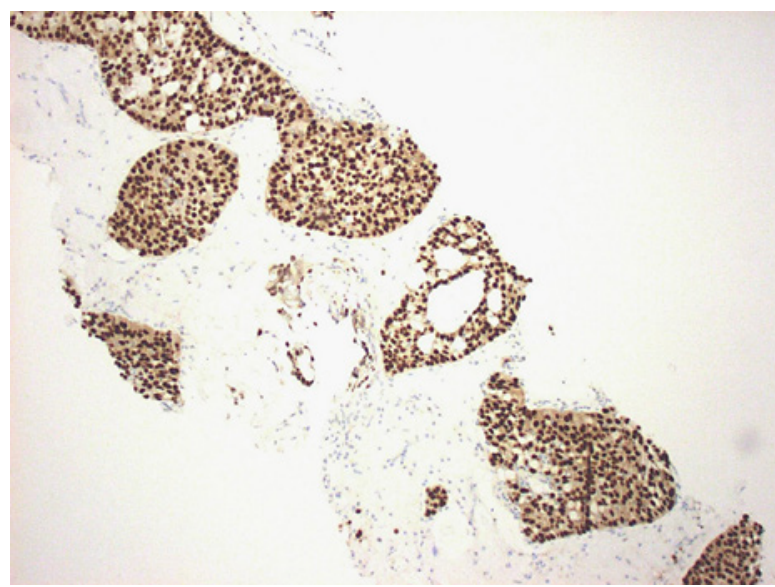

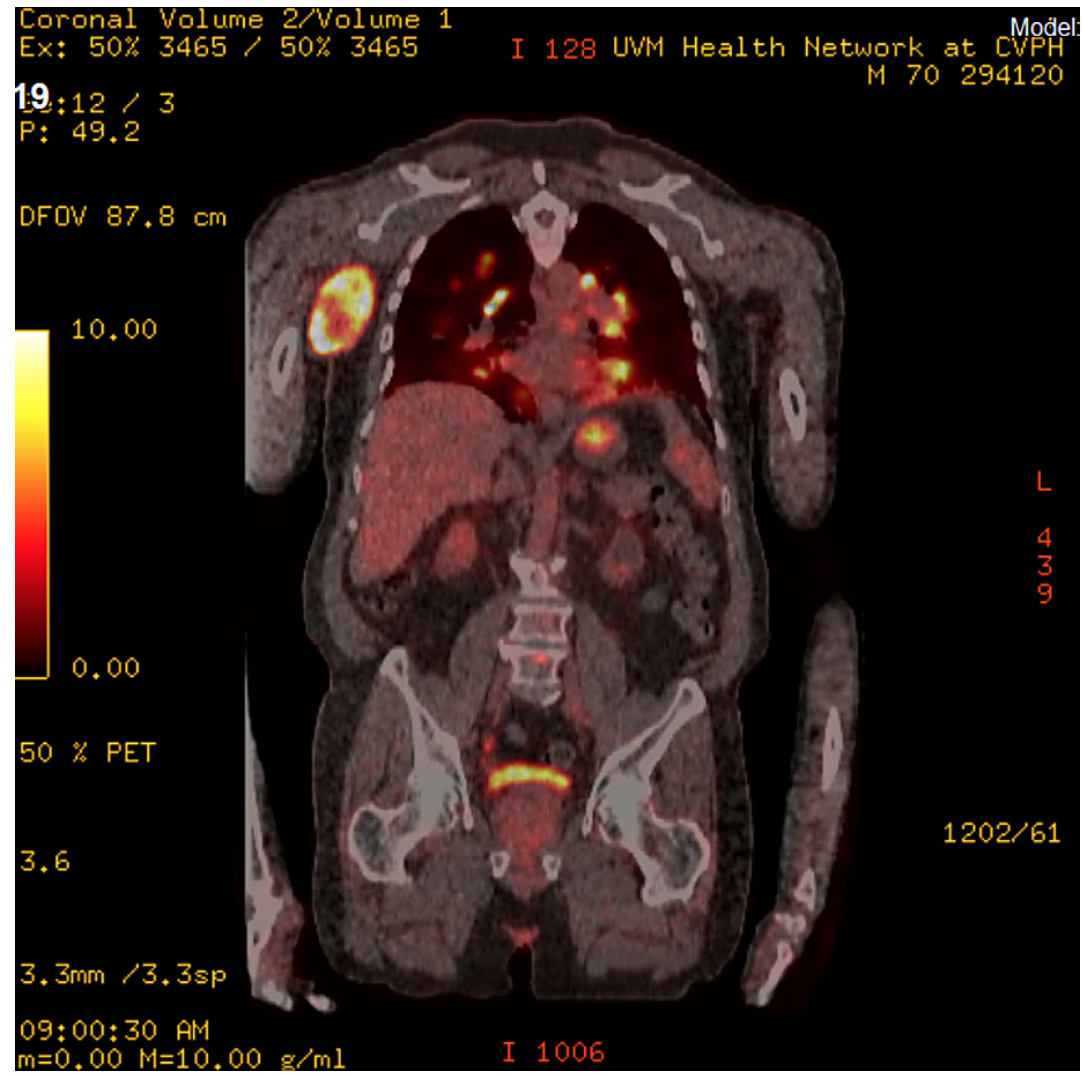

Fig. 6. PET-CT coronal reconstruction image demonstrates innumerable hypermetabolic solid pulmonary nodules with a maximum SUV of 8.3.

increased metabolic activity in either breast. Also, several hypermetabolic small nodules along the margins of the right lobe of the liver, the largest adjacent to the hepatic segment, were found (Fig. 7).

The patient was treated with one cycle of doxorubicin and cyclophosphamide and developed neutropenic fever. He eventually had a bone marrow biopsy and was found to have residual CLL in the marrow. He was switched to palbociclib and anastrozole but again became neutropenic, so he declined further treatment and elected hospice care. 
Fig. 7. Coronal MIPS reconstruction PET image demonstrates multiple foci of avid FDG uptake within the lungs, mediastinum, right axilla, liver, and bone (left iliac crest and L5 vertebral body). There is no abnormal uptake of FDG within the breast soft tissues. Breast cancer in a male with CLL.

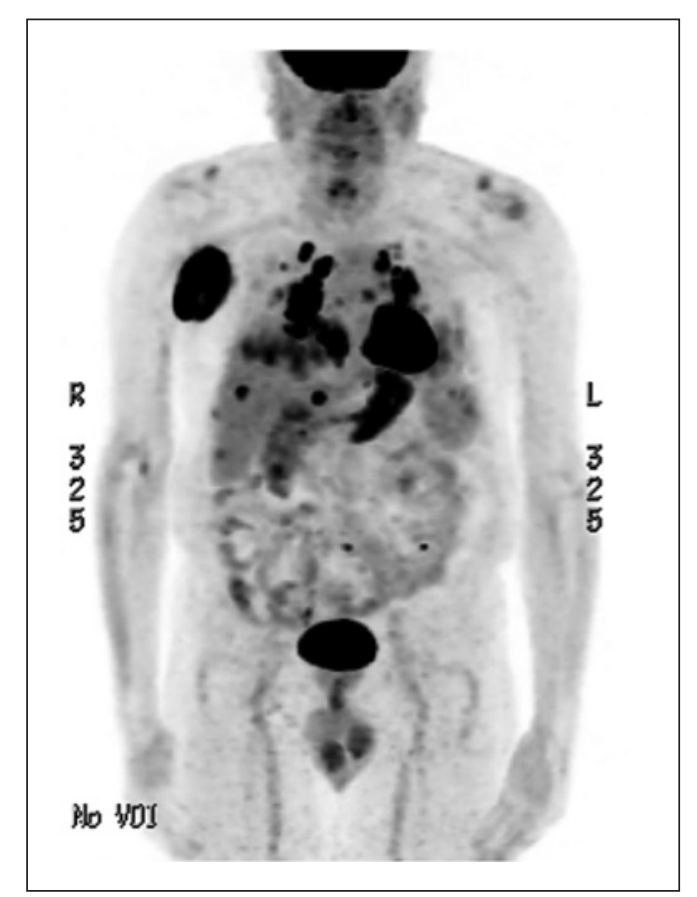

\section{Discussion}

According to SEER data, CLL is the most common leukemia in the Western world, accounting for five per 100,000 men and women per year, with an $86.1 \%$ survival rate. In 2017, an estimated 186,422 people with CLL were living in the USA. Two-thirds of CLL patients require therapy during the disease course. With chemotherapy, most patients achieve complete remission, have long-term survival, and live with their disease for extended periods; thus, they are exposed to complications, infections, autoimmune disorders, and the presence of other cancers during their lifetime [3]. Antineoplastic chemotherapy and immunosuppression caused by CLL itself are considered risk factors for secondary malignancies [4]. Nevertheless, the presence of CLL and carcinoma of the breast in males is infrequent.

\section{CLL and Other Cancers}

Long-term survivors of CLL, especially $>60$-year-old males, are at higher risk of developing other cancers than the general population. Also, studies have shown that the presence of other cancers in these patients may be associated with shorter survival [4]. In a study done by Falchi et al. [4] at the MD Anderson Cancer Center, out of 797 long-term survival CLL patients, with a mean age of 56 years and $57 \%$ being male, 286 had another type of cancer. Out of these, 76 had $>$ two types of cancers, and one patient had six concomitant cancers $-41 \%$ of these patients being diagnosed after treatment for CLL. The most common accompanying kind of cancer in these patients was nonmelanoma skin cancer. A study done by Farma et al. [5] revealed that patients with melanoma had a ten-fold increase of CLL diagnosis than colorectal cancer patients, an eight-fold increase compared to prostate cancer patients, and a four-fold increase compared to breast cancer patients. Excluding the latter, the most common types reported by Falchi et al. [4] were prostate, breast, lung, and GI tumors. Interestingly, out of the 286 patients with other cancers, $<20$ were diagnosed with breast cancer before or concomitantly with CLL, and almost 20 were diagnosed with breast cancer after the CLL diagnosis.

\section{Karger'}


Most importantly and relevant to our report, a study done by Solomon et al. [6] in 2013 reported the incidence of different types of malignancies on CLL and non-CLL patients in a particular population. Out of 579,164 patients, 608 had CLL and developed breast cancer. Out of these 608, zero were males [6]. This information is crucial given the fact that our patient is a male. Because of all of this, we believe our case is unique, as it is not common to see metastatic lung cancer with breast origin, especially in male CLL patients. Patients with CLL have more than twice the risk of developing secondary cancer and an increased frequency of certain types. Awareness of risk factors could permit early detection [7].

According to 2015 SEER data, the incidence of invasive breast cancer in men is 1.1 in 100,000 , whereas in women, it is 126:100,000 [8]. Furthermore, this is a case of occult breast cancer $(\mathrm{OBC})$ which makes this case even more unique, with $\mathrm{OBC}$ being a type of breast cancer where there is no primary cancer lesion found in either physical examination or imaging. Additionally, this is the fifth case report of male OBC reported in the literature, one case of metastatic infiltrating ductal carcinoma in China in 2008, and two other cases of metastatic carcinoma and adenocarcinoma reported in Korea in 2012. However, our case is distinct given its CLL setting and histologic and clinical features [9]. Male breast cancer is more common in African Americans than Hispanics, Caucasians, and Asians [8]. A study performed at the Memorial Sloan Kettering Cancer Center about breast cancer in female versus male patients reported that out of all male breast cancers, approximately $10 \%$ are in situ carcinomas, and the remaining $90 \%$ are infiltrating ductal carcinomas, which was, in fact, our patient's breast cancer type. Infiltrating lobular carcinoma, medullary lesions, and neuroendocrine tumors are rare and have been reported in patients with Klinefelter syndrome. Male breast cancers usually express the ER, PR, and androgen receptor and are hormonally responsive, and our patient was, indeed, ER+ and PR+ [8], with a TNM classification of TxN3M1. Diagnosis of male breast cancer can be made by triple assessment: clinical assessment, radiologic assessment (mammography and ultrasound examination), and tissue biopsy (FNA), just as in female breast cancer [8]. Mammography can identify malignant breast tumors with a sensitivity of $92-100 \%$ and a specificity of 90\%; axillary ultrasound can be helpful in staging [10].

A study done by Nofal and Yousef [10] in 2019 stated that the most common symptom of breast cancer, and usually the first, is a painless retroareolar mass, which is present alone, or with other symptoms in $75 \%$ of all cases. The mass was more frequently found on the left than on the right, and pain was present only in 5\% of the patients [9]. Our patient's breast cancer was reported on the right side rather than on the left, and there were no evident clinical signs, besides the axillary mass, despite the statistics.

Axillary lymph node involvement at presentation is more common in men than in women, as was seen in a progressed state with our patient. Male breast cancer risk factors include advanced age, antiandrogen therapy, radiotherapy, and hormonal imbalance, as well as BRCA-2 mutations $[8,10]$.

\section{Conclusion}

Male breast cancer is rare and represents approximately $1 \%$ of cancers in men and $1 \%$ of cancers worldwide. Because of this low incidence, breast cancer literature, research, and clinical trials focus on females. Even though these may aid in the male counterpart diagnosis, molecular and clinicopathologic features differ between them [8].

In developed countries, almost half of male breast cancers have surpassed local invasion at the time of the diagnosis [8]. This report aims to portray risk factors like concomitant diseases, history of malignancy, and essential clinical characteristics as tools that can aid in the early diagnosis of an otherwise rare disease, as male breast cancer is,

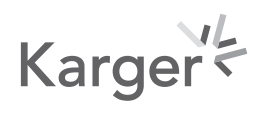


in developed and underdeveloped countries. Lack of information and awareness of this disease can lead to its progression and devastating death. We hope this case report was helpful to health care providers, as they must be informed on the different manifestations of male breast cancer.

\section{Acknowledgment}

Thanks are due to Jill Tarabula, MLS, AHIP, for her help in the technical editing of this manuscript.

\section{Statement of Ethics}

The study is exempt from ethics committee approval. It is a retrospective case report. Written informed consent was obtained from the patient's substitute decision maker for publication of this case report and any accompanying images.

\section{Conflict of Interest Statement}

The authors have no conflicts of interest to declare.

\section{Funding Sources}

This research did not receive grants from any funding agency in the public, commercial, or not-for-profit sectors.

\section{Author Contributions}

Jose M. Acostamadiedo, MD, contributed to conceptualization, methodology, software, reviewing and editing, and supervision. Ana S. Acostamadiedo Marx and Camilo Andrés Avendaño-Capriles, MS, contributed to data curation and writing - original draft preparation. Julio Lemos, MD, contributed to visualization and investigation. Eric Gorman, MD, contributed to visualization and investigation.

\section{Data Availability Statement}

Data are not publicly available in accordance with HIPAA.

\section{References}

1 Barroeta JE, Farkas T. Merkel cell carcinoma and chronic lymphocytic leukemia (collision tumor) of the arm: a diagnosis by fine-needle aspiration biopsy. Diagn Cytopathol. 2007;35(5):293-5.

2 Dubashi B, Jain A, Srinivasan K, Surendrakumar V, Vivekanandam S. Chronic lymphocytic leukemia and breast cancer as synchronous primary in a male-a rare combination. Curr Oncol. 2011;18(2):e101-2.

3 National Cancer Institute. Cancer stat facts: Leukemia - chronic lymphocytic leukemia (CLL). National Institutes of Health; 2021. https://seer.cancer.gov/statfacts/html/clyl.html. 
4 Falchi L, Vitale C, Keating MJ, Lerner S, Wang X, Elhor Gbito KY, et al. Incidence and prognostic impact of other cancers in a population of long-term survivors of chronic lymphocytic leukemia. Ann Oncol. 2016;27(6): 1100-6.

5 Farma JM, Zager JS, Barnica-elvir V, Puleo CA, Marzban SS, Rollison DE, et al. A collision of diseases: Chronic lymphocytic leukemia discovered during lymph node biopsy for melanoma. Ann Surg Oncol. 2013;20:1360-4

6 Solomon B, Rabe K, Slager S, Brewer J, Cerhan J Shanafelt T. Overall and cancer-specific survival of patients with breast, colon, kidney, and lung cancers with and without chronic lymphocytic leukemia: a SEER population-based study. J Clin Oncol. 2013;31(7):930-7.

7 Tsimberidou AM, Wen S, McLaughlin P, O'Brien S, Wierda WG, Lerner S, et al. Other malignancies in chronic lymphocytic leukemia/small lymphocytic lymphoma. J Clin Oncol. 2009;27(6):904-10.

8 Gucalp A, Traina TA, Eisner JR, Parker JS, Selitsky SR, Park BH, et al. Male breast cancer: a disease distinct from female breast cancer. Breast Cancer Res Treat. 2019;173:37-48.

9 He M, Liu H, Jiang Y. A case report of male occult breast cancer first manifesting as axillary lymph node metastasis with part of metastatic mucinous carcinoma. Medicine. 2015 Jun;94(25):e1038.

10 Nofal MN, Yousef AJ. The diagnosis of male breast cancer. Neth J Med. 2019;77(10):356-9. http://www. njmonline.nl/getpdf.php?id=2169. 